\title{
Pattern Synthesis of Time-Modulated Array Antenna Based on an Improved Invasive Weed Optimization Method
}

\author{
Zhenkai Zhang $\mathbb{D}^{1,2}$ Xinxing Liu, ${ }^{1}$ Bing Zhang, ${ }^{1}$ and Hailin $\mathrm{Li}^{3}$ \\ ${ }^{1}$ College of Electronics Information, Jiangsu University of Science and Technology, Zhenjiang, China \\ ${ }^{2}$ Department of Electrical and Computer Engineering, University of Calgary, Calgary, Canada \\ ${ }^{3}$ Key Laboratory of Radar Imaging and Microwave Photonics, Nanjing University of Aeronautics and Astronautics, Nanjing, China
}

Correspondence should be addressed to Zhenkai Zhang; zhangzhenkai@just.edu.cn

Received 1 June 2018; Accepted 7 February 2019; Published 2 May 2019

Academic Editor: Sotirios K. Goudos

Copyright ( 2019 Zhenkai Zhang et al. This is an open access article distributed under the Creative Commons Attribution License, which permits unrestricted use, distribution, and reproduction in any medium, provided the original work is properly cited.

In this paper, pattern synthesis through time-modulated linear array is studied, and a novel strategy for harmonic beamforming in time-modulated array is proposed. The peak side lobe level is designed as optimization objective function, and the switch-on time sequence of each element is selected as optimization variable. An improved invasive weed optimization (IWO) algorithm is developed in order to determine the optimal parameters describing the pulse sequence used to modulate the excitation weights of array elements. Representative results are reported and discussed to point out potentialities and advantages of the proposed approach, which can obtain lower objective function values.

\section{Introduction}

With the rapid development of radar and electronic technology, the requirement for better performance of the array antenna is continuing to rise up. Pattern synthesis of antenna arrays is one of the important issues in antenna array design. The essence of pattern synthesis is to determine some parameters of antenna array so that the rational pattern can conform to the desired pattern as far as possible. In the synthesis of ultralow side lobe pattern, it is difficult to obtain the desired pattern by adjusting the amplitude and phase of the element with the conventional antenna arrays. The advantage of time-modulated array lies in beamforming, which is achieved with switches instead of phase shifters. Radio frequency (RF) switches based on semiconductors can be low-cost and high-power-handling components operating in high-frequency range. The initial application of time-modulated antenna array is the pattern synthesis of ultralow side lobe, but the side effect is the harmonic radiation. Some researches on suppressing harmonic radiation level were proposed in $[1,2]$. A novel approach based on the differential evolution (DE) algorithm is proposed to suppress the sideband radiation patterns in time-modulated linear antenna arrays in [1], then the sideband level of a time-modulated linear array can be reduced significantly by rearranging the static excitation amplitudes as well as the switch-on time intervals of each element. An innovative approach for the synthesis of timemodulated linear array (TMLA) antennas is presented in [3], which uses the particle swarm optimization to fully exploit the dependence of the sideband radiations (SR) on the shift of the time pulses. The synthesis of simultaneous multiple beams through time-modulated linear arrays is studied in [4], and the periodic on-off sequences controlling the static excitations are properly defined by means of an optimization strategy based on the particle swarm algorithm. The variation of side lobe level of optimized uniformly excited time-modulated linear antenna array with different number of antenna elements is presented in [5], and a differential evolution-based optimization method is employed where only the on-time sequence of the antenna elements is considered as the optimization parameter vectors. The paper [6] presents experimental results using time modulation to implement inexpensive side lobe blanking. 
The paper [7] proposes to time-modulate the array excitations with periodic sum-of-weighted-cosine pulses rather than with the periodic rectangular pulses. Although these pattern synthesis methods have been developed, the beamforming for time-modulated array does not have a considerable side lobe. In order to get the best beamforming performance, the amplitude and phase of harmonic need to be optimized for controlling the switch-on time sequence of each element.

Invasive weed optimization is a global stochastic optimization algorithm which was proposed by Mehrabian and Lucas in 2006 [8]. IWO algorithm generates the new seeds around the parent by using the normal diffusion mechanism. It has been widely used in many fields, such as the adaptive beamforming of antenna [9], the optimization of MIMO antenna array [10], overcurrent relay coordination [11], electromagnetics [12], network optimization [13], and resource planning of residential complex energy system [14]. Compared with other algorithms, it has larger search space, but it is inferior to other intelligent algorithms in depth of search. However, the IWO algorithm converges slowly and it tends to trap in early convergence when entering the late stage.

In this paper, an improved algorithm is proposed, in which the individual with strong adaptability in the parent individuals is selected and the seeds generated by this individual are distributed with updating formula of position and velocity instead of normal diffusion. Then the improved IWO algorithm is used in the beamforming of time-modulated linear array. In order to validate the feasibility and effectiveness of the improved algorithm, simulation experiments of the improved algorithm are carried out.

The paper is organized as follows. The mathematical theory of the problem is formulated in Section 2, whereas the optimization strategy is described in Section 3. Selected numerical results are presented and discussed in Section 4. Some conclusions are finally remarked in Section 5.

\section{Mathematical Model of Time-Modulated Antenna Array}

2.1. Basic Theory of Time-Modulated Antenna Array. Considering a linear array of $N$ isotropic elements, the element spacing is half of the wavelength and the radiation pattern is given by

$$
F(\theta)=\sum_{n=0}^{N-1} I_{n} e^{j n \beta d \sin \theta}
$$

where $\beta=2 \pi / \lambda$ is the wavenumber, $d$ is the element spacing, $I_{n}$ is the static excitation amplitude of the $n$th element, and $\theta \in[-\pi / 2, \pi / 2]$.

The structure of time-modulated antenna array is given in Figure 1.

Each element is connected with a radio frequency (RF) switch and the time sequence of each RF switch can be controlled by a digital complex programmable logic device. It is

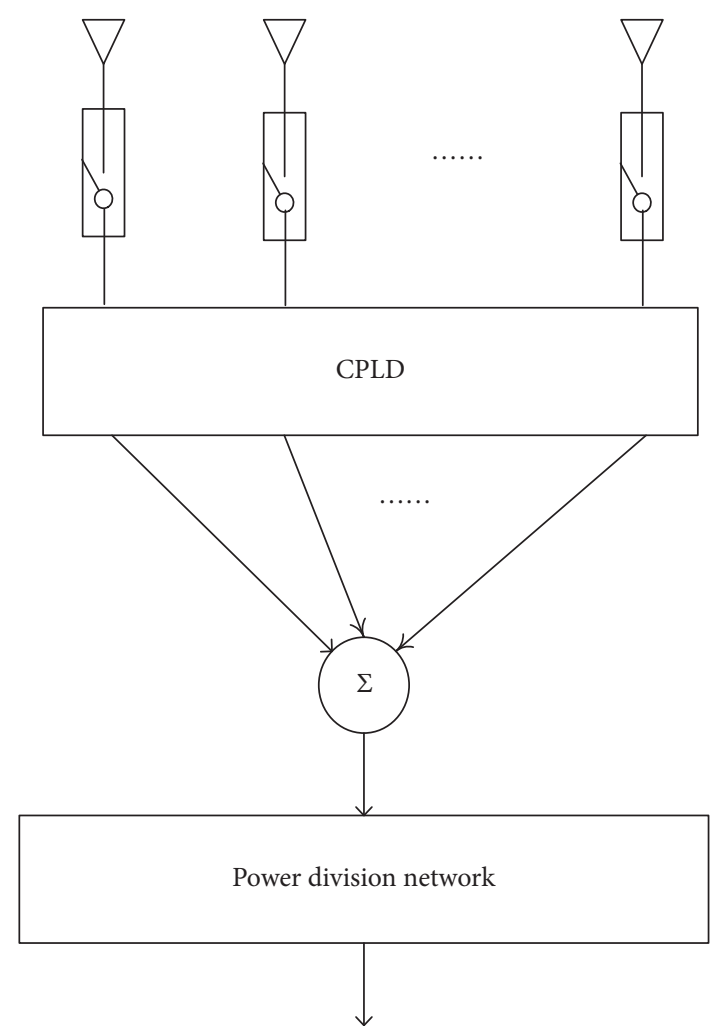

FIgURE 1: Basic structure of time-modulated antenna array.

equal to the antenna signal which is modulated by a periodic signal. Thus, (1) can be rewritten as

$$
F(\theta)=\sum_{n=0}^{N-1} I_{n} U_{n}(t) e^{j n \beta d \sin \theta}
$$

where $U_{n}(t)$ is the periodic signal of $n$th element, as shown in Figure 2, and it can be written as

$$
U_{n}(t)= \begin{cases}1, & t_{\mathrm{on}, n} \leq t \leq t_{\mathrm{on}, n}+t_{d, n} \\ 0, & \text { otherwise }\end{cases}
$$

where $t_{\text {on }}$ is the instant time of the switch opening and $t_{d}$ is the duration of the switch closure time. According to the Fourier transform, $U_{n}(t)$ can be decomposed into

$$
U_{n}(t)=\sum_{k=-\infty}^{\infty} c_{n k} e^{j 2 \pi k f_{m} t}
$$

where $f_{m}=1 / T_{m}$ is the modulation frequency; $c_{n k}$ is the complex Fourier coefficient of $k$ th harmonic which can be expressed as

$$
c_{n k}=\frac{\sin \left(\pi k f_{m} t_{d}\right)}{\pi k} e^{-j k \pi f_{m}\left(2 t_{\mathrm{on}, n}+t_{d, n}\right)} .
$$




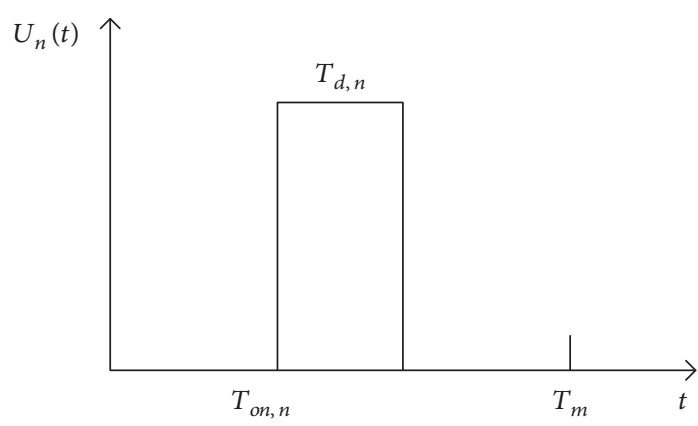

Figure 2: The switch-on time sequence of $n$th element.

In summary, the element factors of the fundamental and harmonic components can be calculated as

$$
\begin{aligned}
F_{0}(\theta) & =\sum_{n=0}^{N-1} I_{n} v_{n} e^{j n \beta d \sin \theta}, \\
F_{1}(\theta) & =\sum_{n=0}^{N-1} I_{n} \sin \left(\pi v_{n}\right) e^{-j \pi\left(2 \mu_{n}+v_{n}\right)} e^{j n \beta d \sin \theta}, \\
F_{-1}(\theta) & =\sum_{n=0}^{N-1} I_{n} \sin \left(\pi v_{n}\right) e^{j \pi\left(2 \mu_{n}+v_{n}\right)} e^{j n \beta d \sin \theta},
\end{aligned}
$$

where

$$
\left\{\begin{array}{l}
\mu_{n}=f_{m} t_{\mathrm{on}, n}, \\
v_{n}=f_{m} t_{d, n} .
\end{array}\right.
$$

By analyzing (7) and (8), it can be concluded that $F_{1}(\theta)$ and $F_{-1}(\theta)$ are symmetrical about the normal direction of the antenna array. The range of $v_{n}$ should be $[0,0.5]$ to make excitation amplitude varied in the range $[0,1]$. Analogously, in order to make the phase shift range from $[-\pi, \pi], \mu_{n}$ should vary in the range $[-0.5,0.5]$.

Suppose $A_{n}$ is the element excitation amplitude obtained by Chebyshev synthesis and $\theta_{0}$ is the beam pointing angle of the positive first harmonic component, $v_{n}$ and $\mu_{n}$ can be calculated by

$$
\left\{\begin{array}{l}
v_{n}=1 / \pi \cdot \arcsin \left(A_{n} / I_{n}\right), \\
\mu_{n}=1 / 2\left(\left[\left(n \beta d \sin \theta_{0}\right) / \pi-v_{n}\right]\right) .
\end{array}\right.
$$

In conclusion, the desired patterns can be obtained by controlling the switch-on time sequence of each element.

The peak side lobe level of radiation pattern can be expressed as

$$
\left\{\begin{array}{l}
\operatorname{PSLL}_{0}=\max \left(\left|F_{0}(\theta)\right|_{\theta \in S_{0}}\right), \\
\operatorname{PSLL}_{1}=\max \left(\left|F_{1}(\theta)\right|_{\theta \in S_{1}}\right), \\
\operatorname{PSLL}_{-1}=\max \left(\left|F_{-1}(\theta)\right|_{\theta \in S_{1-}}\right)
\end{array},\right.
$$

where $S_{0}, S_{1}$, and $S_{2}$ are the side lobe regions of the corresponding radiation pattern, respectively. The objective function is set to

$$
f(x)=\alpha\left(\mathrm{PSLL}_{0}-\mathrm{SLL}\right)+\beta\left(\mathrm{PSLL}_{1}-\mathrm{SLL}\right),
$$

where SLL is the specified side lobe level value; $\alpha$ and $\beta$ are the corresponding weighting factors, respectively.

2.2. Chebyshev Synthesis. The Chebyshev synthesis method was put forward to approximate the array factor function, obtaining the method of Chebyshev synthesis. The $m$-order Chebyshev polynomial $T_{m}(x)$ is the solution of the following second-order differential equation:

$$
\left(1-x^{2}\right) \frac{d^{2} T_{m}(x)}{d x^{2}}-x \frac{d T_{m}(x)}{d x}+m^{2} T_{m}(x)=0 .
$$

$T_{m}(x)$ can be expressed as

$$
T_{m}(x)= \begin{cases}\cos \left(m \cos ^{-1}(x)\right), & |x| \leq 1, \\ \cosh \left(m \cosh ^{-1}(x)\right), & |x|>1 .\end{cases}
$$

The array factor of an $N$ element uniform linear array can be expressed as

$$
F(\theta)= \begin{cases}\sum_{n=0}^{(N-1) / 2} I_{n} \cos \left((2 n) \frac{u}{2}\right), & N \text { is an odd number } \\ \sum_{n=0}^{(N / 2)-1} I_{n} \cos \left((2 n+1) \frac{u}{2}\right), & N \text { is an even number }\end{cases}
$$

where $u=2 \pi d \sin \theta / \lambda$. It can be found that the expression of the array factor is similar to the Chebyshev polynomial by comparing (14) and (15). Thus, the array factor can be equated to the form of Chebyshev polynomial.

\section{Invasive Weed Optimization}

3.1. Description of Invasive Weed Optimization Process for the Beamforming. The IWO algorithm is a random search algorithm derived from the evolution principle of weeds in nature, and it is an algorithm that mimics the invasion process of weeds. It has been proven to be very robust and adaptable. The algorithm steps are as follows:

(1) The element excitation amplitude can be obtained by Chebyshev synthesis. $v_{n}$ and $\mu_{n}$ are calculated by (10)

(2) $v_{n}$ is selected as an optimization variable and the initial population is $X=\left(X_{1}, X_{2}, \ldots, X_{N}\right)$, where $N$ is the number of elements. $F_{0}(\theta), F_{1}(\theta)$, and $F-{ }_{1}(\theta)$ are calculated by (7)-(9). The objective function value can be obtained by (11) and (12) 


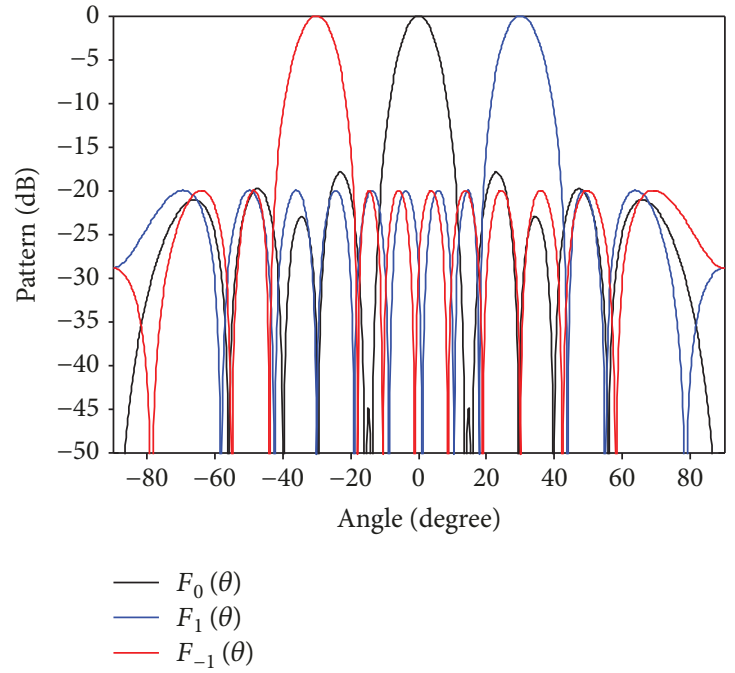

(a) $\theta_{0}=30^{\circ}$

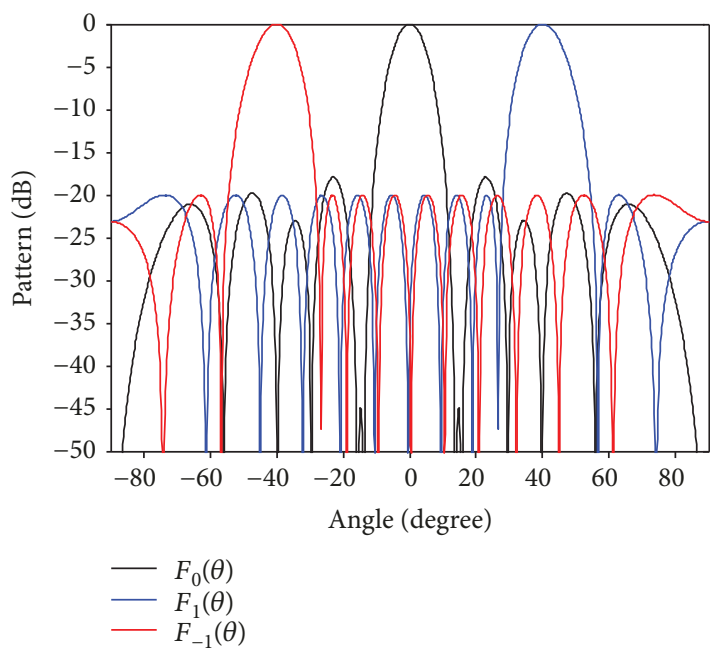

(b) $\theta_{0}=40^{\circ}$

FIgURE 3: Rational patterns obtained by Chebyshev synthesis.

(3) $f_{\max }$ and $f_{\min }$ are the maximum and minimum values of the objective function, respectively. The number of seeds that each individual can produce is calculated by

$$
\text { weed }_{t}=\operatorname{ceil}\left(\frac{f-f_{\text {min }}}{f_{\text {max }}-f_{\text {min }}}\left(s_{\text {max }}-s_{\text {min }}\right)+s_{\text {min }}\right),
$$

where $s_{\max }$ and $s_{\min }$ are the maximum and minimum number of seeds that each individual can produce

(4) The generated seeds are randomly distributed around the parent individuals by normal distribution mechanism in which the mean is zero and the variance is $\sigma^{2}$. The standard deviation $\sigma$ can be calculated by

$$
\sigma_{\text {iter }}=\sigma_{\min }+\left(\frac{\text { iter }_{\max }-\text { iter }}{\text { iter }_{\max }}\right)^{\omega}\left(\sigma_{\max }-\sigma_{\min }\right)
$$

where $\sigma_{\max }$ and $\sigma_{\min }$ are the maximum and minimum standard deviation, iter ${ }_{\max }$ and iter are the maximum iteration number and current iteration number, respectively, and $\omega$ is the nonlinear regulatory factor

(5) If the number of population exceeds the maximum number of population $P_{\max }$, all individuals are sorted from the largest to the smallest according to the value of objective function. Only the first $P_{\max }$ individuals which are selected from the sorted individuals can be preserved as new population. Steps 2 to 4 will repeat until the number of iteration reaches iter $_{\max }$

3.2. Improved Procedure of Invasive Weed Optimization. In order to improve the local optimization ability of the IWO algorithm, the updating of position and velocity is introduced into the seeds generated by the parent individual. In the process of seed spatial distribution, the individual $\left(X_{\text {best }}\right)$ with the maximum value of the objective function is selected. The updating formula of position and velocity is given by

$$
\left\{\begin{array}{l}
v_{i}(k+1)=\omega v_{i}(k)+c_{1} r_{1}\left(p_{i}(k)-x_{i}(k)\right)+c_{2} r_{2}\left(p_{g}-x_{i}(k)\right), \\
x_{i}(k+1)=x_{i}(k)+v_{i}(k+1),
\end{array}\right.
$$

where $c_{1}$ and $c_{2}$ are learning factors, $r_{1}$ and $r_{2}$ are random numbers in the range of $[0,1], p_{i}(k)$ is the best position of the $k$ th particle in the "flying" history, $p_{g}$ is the best position of all particle, and $\omega$ is the inertia weight and it can be calculated by

$$
\omega_{\text {iter }}=\omega_{\max }-\frac{\omega_{\max }-\omega_{\min }}{\text { iter }_{\max }} * \text { iter }
$$

where $\omega_{\max }$ is the maximum inertia weight and $\omega_{\min }$ is the minimum inertia weight.

\section{Simulation}

4.1. Simulation Experiment 1. In order to verity the effectiveness of the proposed algorithm, a linear array of 12 array elements is used. Firstly, the simulation experiment of Chebyshev synthesis is carried out and a $-20 \mathrm{~dB}$ SLL Chebyshev pattern is generated. The simulation results are shown in Figure 3. $F_{0}$ is the fundamental pattern; $F_{1}$ and $F_{-1}$ are the harmonic patterns. We can see that $F_{1}$ has a main lobe at $30^{\circ}$ and $40^{\circ}$, respectively.

Figures $3(\mathrm{a})$ and $3(\mathrm{~b})$ are rational patterns that show $\theta_{0}=30^{\circ}$ and $\theta_{0}=40^{\circ}$. The values of $\mu$ and $\nu$ are shown in Tables 1 and 2 . 
TABLE 1: The values of $\mu$ and $v\left(\theta_{0}=30^{\circ}\right)$.

\begin{tabular}{cccccccccccccc}
\hline & 1 & 2 & 3 & 4 & 5 & 6 & 7 & 8 & 9 & 10 & 11 & 12 \\
\hline$\mu$ & -0.13 & 0.16 & 0.37 & -0.41 & -0.20 & 0 & 0.25 & -0.45 & -0.16 & 0.12 & 0.41 & -0.38 \\
$\nu$ & 0.25 & 0.19 & 0.25 & 0.32 & 0.40 & 0.50 & 0.50 & 0.40 & 0.32 & 0.25 & 0.19 & 0.25 \\
\hline
\end{tabular}

TABle 2: The values of $\mu$ and $v\left(\theta_{0}=40^{\circ}\right)$.

\begin{tabular}{cccccccccccccc}
\hline & 1 & 2 & 3 & 4 & 5 & 6 & 7 & 8 & 9 & 10 & 11 \\
\hline$\mu$ & -0.13 & 0.22 & -0.48 & -0.20 & 0.09 & 0.36 & -0.32 & 0.05 & 0.41 & -0.23 & 0.12 & 0.41 \\
$\nu$ & 0.25 & 0.19 & 0.25 & 0.32 & 0.40 & 0.50 & 0.50 & 0.40 & 0.32 & 0.25 & 0.19 & 0.25 \\
\hline
\end{tabular}

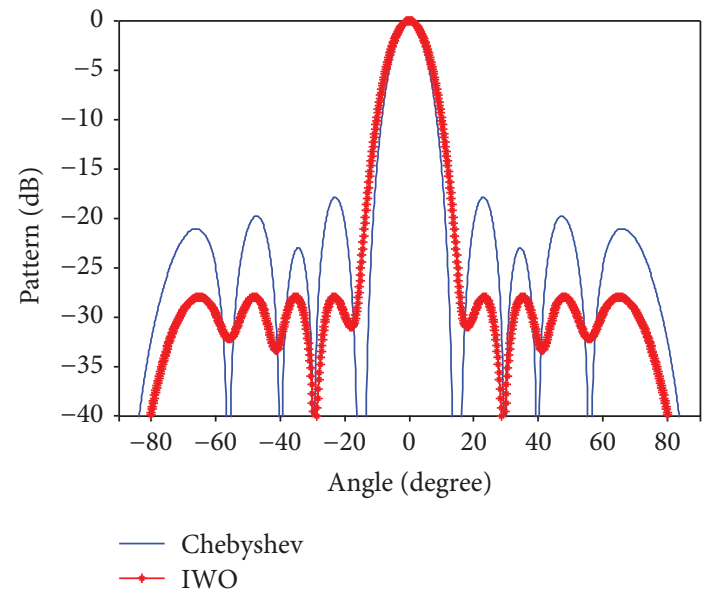

(a) Fundamental patterns

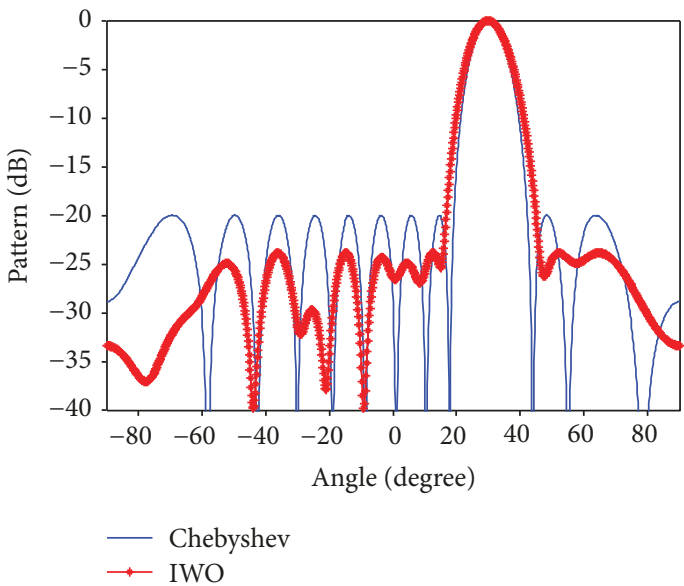

(b) Harmonic patterns

FIGURE 4: Rational patterns obtained by the IWO algorithm.

TABLE 3: The parameters of the IWO algorithm.

\begin{tabular}{lcc}
\hline Parameter & Significance & Value \\
\hline$N P$ & Number of initial population & 10 \\
$N P_{\max }$ & Maximum number of population & 50 \\
$s_{\max }$ & Maximum number of seeds & 1 \\
$s_{\min }$ & Minimum number of seeds & 20 \\
$\sigma_{\min }$ & Initial value of standard deviation & 0.003 \\
$\sigma_{\max }$ & The final value of standard deviation & 0.000003 \\
$\omega$ & Nonlinear regulatory factor & 3 \\
iter $_{\max }$ & Maximum number of iteration & 300 \\
\hline
\end{tabular}

It can be found that the values of $v$ are the same when the beam pointing angle was 30 degrees and 40 degrees by observing the data in Tables 1 and 2. So it can be concluded that the beam pointing angle is only influenced by parameter $\mu$.

4.2. Simulation Experiment 2. It is proved that parameter $v$ is not related to the beam pointing angle in the simulation experiment 1 , so $v$ is taken as optimization variable to suppress the SLLs of the radiation pattern at the
TABLE 4: The parameters of the PSO algorithm.

\begin{tabular}{lcc}
\hline Parameter & Significance & Value \\
\hline$N P$ & Number of population & 100 \\
$v_{\max }$ & Maximum velocity & 0.5 \\
$v_{\min }$ & Minimum velocity & -0.5 \\
$c_{1}, c_{2}$ & Learning factors & 2 \\
$\omega_{\max }$ & Maximum inertia weight & 1 \\
$\omega_{\min }$ & Minimum inertia weight & 0 \\
iter $_{\max }$ & Maximum number of iteration & 300 \\
\hline
\end{tabular}

fundamental and harmonic components. The simulation results are as follows.

As shown in Figure 4, the SLLs optimized by IWO algorithm are lower than Chebyshev synthesis. In order to further confirm the effectiveness of the algorithm, the PSO algorithm is simulated and compared with the IWO algorithm. The parameters of the two algorithms are shown in Tables 3 and 4 . The simulation results are shown in Figure 5.

The simulation results show that the pattern optimized by the IWO algorithm can obtain lower SLLs than the PSO 


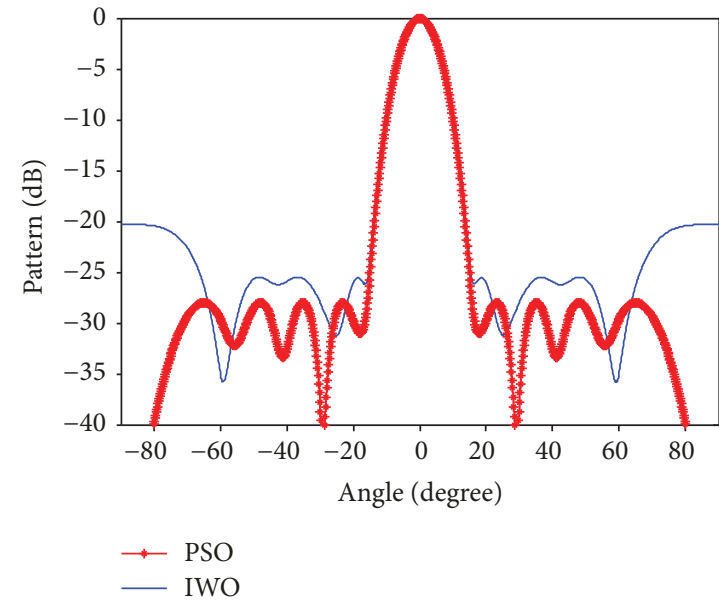

(a) Fundamental pattern

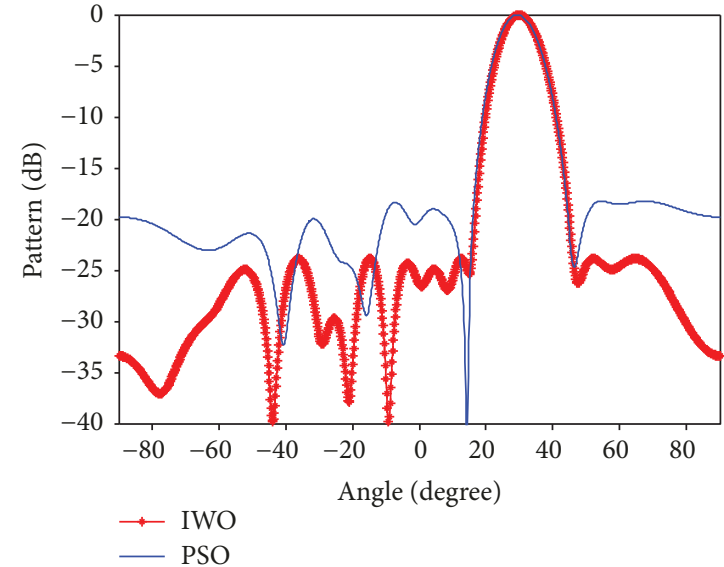

(b) Harmonic pattern

FIgURE 5: Rational patterns obtained by the IWO and PSO algorithms.

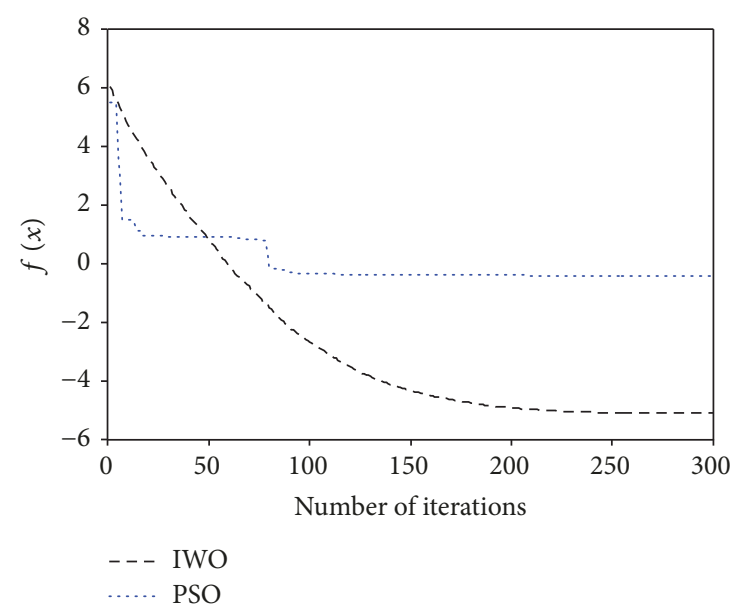

FIGURE 6: Diagram of iterative curves.

algorithm when the widths of main lobes are basically the same. The iterative curves of the two algorithms are shown in Figure 6 and it can be found that the value of the objective function obtained by the IWO algorithm is lower than that by the PSO algorithm. Thus, an improved algorithm combining the PSO algorithm with the IWO algorithm is proposed in this paper, and it is applied to optimize the SLLs of rational pattern. The simulation results are shown in Figure 7.

Figure 8 shows the time sequences of each element in a normalized time modulation period optimized by the improved algorithm, and the shadow parts represent that the switch is closed. The rational patterns show that the SLLs optimized by the improved algorithm at the fundamental are lower than those by the IWO algorithm while the optimization effect of SLLs at harmonic is not desired. For multiobjective optimization problem, the linear weighted sum method is used in this paper and some further improvement researches need to be done. The iterative curves of the three algorithms are shown in Figure 9. It can be found that the proposed optimization method is most effective with the lowest fitness value, as the convergence speed of the PSO

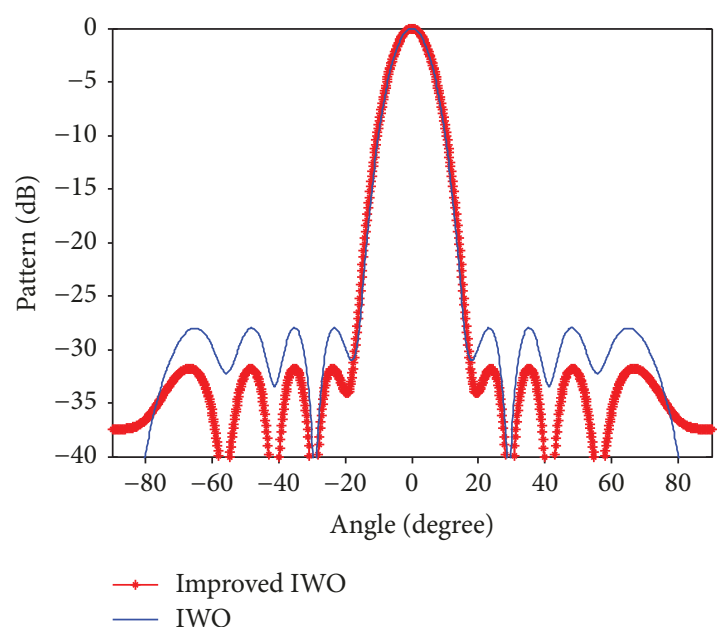

(a) Fundamental pattern

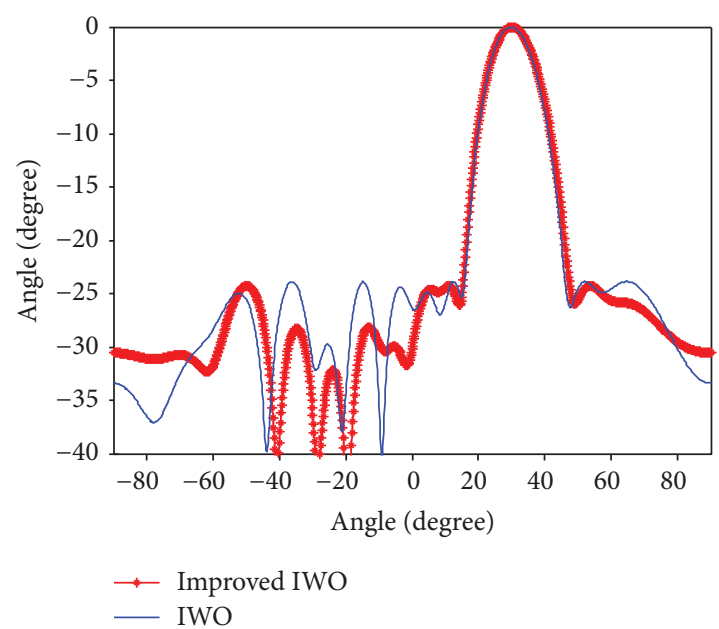

(b) Harmonic pattern

FIgURE 7: Rational patterns obtained by the IWO and PSO-IWO algorithms. 


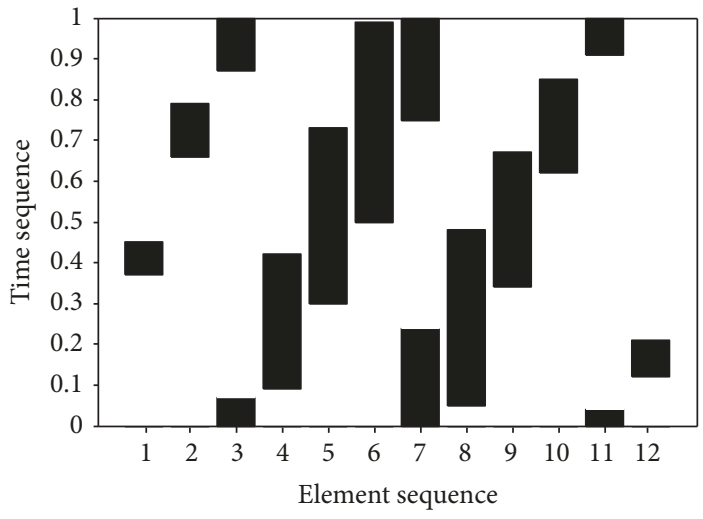

FIgURE 8: Time sequences of elements.

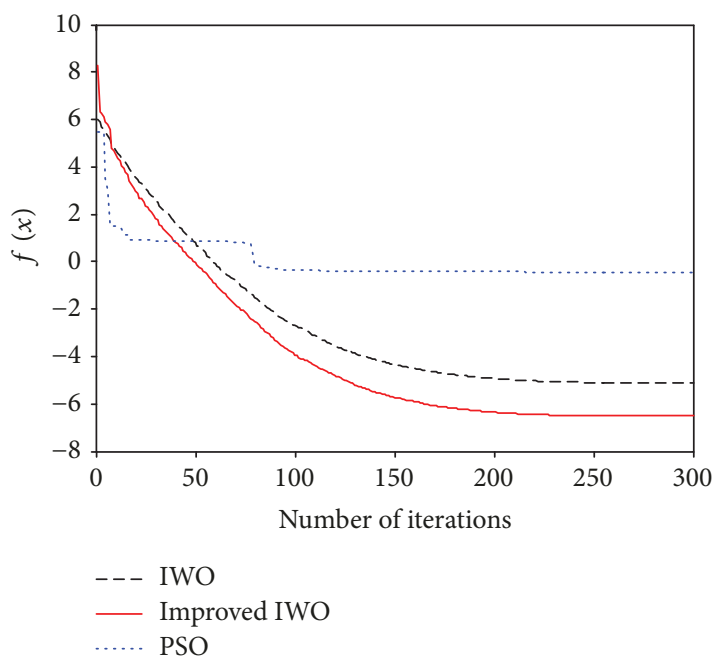

FIgURE 9: Diagram of iterative curves.

algorithm is fast but easy to trap in local optimum and the optimal effect of the IWO algorithm is better than the PSO algorithm due to the slower convergence speed. In the improved IWO algorithm, the updating of position and velocity is introduced into the seeds generated by the parent individual; the individual with strong adaptability in the parent individuals is selected.

The values of PSLL and $f(x)$ are shown in Table 5. It can be clearly seen that the performance of the proposed algorithm has a lower PSLL and deeper null steering in the desired direction.

\section{Conclusion}

An improved IWO algorithm was used in pattern synthesis based on time-modulated antenna array in this paper. In order to get radiation patterns with low side lobe levels, the switch-on time sequence of each element was selected as the optimal variable. The simulation results showed that the SLLs optimized by the improved IWO were lower than those optimized by other algorithms. It indicated that the improved IWO was effective. However, there are still some
TABLE 5: The values of PSLL and $f(x)$.

\begin{tabular}{lcccc}
\hline & $\begin{array}{c}\text { Element } \\
\text { number }\end{array}$ & $\operatorname{PSLL}_{0}(\mathrm{~dB})$ & $\operatorname{PSLL}_{1}(\mathrm{~dB})$ & $f(x)$ \\
\hline Chebyshev & 12 & -17.90 & -20 & Null \\
PSO & 12 & -25.49 & -18.26 & -0.43 \\
IWO & 12 & -27.99 & -23.88 & -5.11 \\
Improved IWO & 12 & -31.77 & -24.27 & -6.52 \\
\hline
\end{tabular}

deficiencies to improve. For the problem of multiobjective optimization, further research is still needed. The more indepth research about how to optimize multiobjective functions will be done in the future.

\section{Data Availability}

There are no data sharing issues since all of the numerical information is produced by solving the equations of the proposed algorithm, which are realized by MATLAB software in the paper. The simulation data and programs used to support the findings of this study are available from the corresponding author upon request.

\section{Conflicts of Interest}

The authors declare that there is no conflict of interest regarding the publication of this paper.

\section{Acknowledgments}

This work was supported by the National Natural Science Fund $(61401179,61671239)$ in China, Postgraduate Research \& Practice Innovation Program of Jiangsu Province (SJCX18_0766), China Postdoctoral Science Foundation (2016M592334), and Qing Lan Project of Jiangsu Province.

\section{References}

[1] S. Yang, Y. B. Gan, and A. Qing, "Sideband suppression in time-modulated linear arrays by the differential evolution algorithm," IEEE Antennas and Wireless Propagation Letters, vol. 1, pp. 173-175, 2002.

[2] S. Yang, Y. B. Gan, A. Qing, and P. K. Tan, "Design of a uniform amplitude time modulated linear array with optimized time sequences," IEEE Transactions on Antennas and Propagation, vol. 53, no. 7, pp. 2337-2339, 2005.

[3] L. Poli, P. Rocca, L. Manica, and A. Massa, "Pattern synthesis in time-modulated linear arrays through pulse shifting," IET Microwaves, Antennas \& Propagation, vol. 4, no. 9, pp. 1157-1164, 2010.

[4] L. Poli, P. Rocca, G. Oliveri, and A. Massa, "Harmonic beamforming in time-modulated linear arrays," IEEE Transactions on Antennas and Propagation, vol. 59, no. 7, pp. 25382545, 2011.

[5] S. K. Mandal, H. Singh, A. Mukherjee, and K. Mandal, "A study on variation of side lobe level of optimized uniformly excited time-modulated linear antenna arrays," in 2017 11th European Conference on Antennas and Propagation (EUCAP), Paris, France, March 2017. 
[6] W. C. Barott and S. Fucharoen, "Experimental timemodulated beamformer for interference mitigation in a radio spectrometer," IEEE Journal of Selected Topics in Signal Processing, vol. 11, no. 2, pp. 271-281, 2017.

[7] R. Maneiro-Catoira, J. C. Brégains, J. A. García-Naya, and L. Castedo, "Enhanced time-modulated arrays for harmonic beamforming," IEEE Journal of Selected Topics in Signal Processing, vol. 11, no. 2, pp. 259-270, 2017.

[8] A. R. Mehrabian and C. Lucas, "A novel numerical optimization algorithm inspired from weed colonization," Ecological Informatics, vol. 1, no. 4, pp. 355-366, 2006.

[9] M. M. Roshanaei, C. Lucas, and A. R. Mehrabian, “Adaptive beamforming using a novel numerical optimisation algorithm," IET Microwaves, Antennas \& Propagation, vol. 3, no. 5, pp. 765-773, 2009.

[10] S. Karimkashi, A. A. Kishk, and D. Kajfez, "Antenna array optimization using dipole models for MIMO applications," IEEE Transactions on Antennas and Propagation, vol. 59, no. 8, pp. 3112-3116, 2011.

[11] C. A. Castillo, A. Conde, and M. Y. Shih, "Improvement of non-standardized directional overcurrent relay coordination by invasive weed optimization," Electric Power Systems Research, vol. 157, pp. 48-58, 2018.

[12] S. Karimkashi and A. A. Kishk, "Invasive weed optimization and its features in electromagnetics," IEEE Transactions on Antennas and Propagation, vol. 58, no. 4, pp. 1269-1278, 2010.

[13] D. Rama Prabha and T. Jayabarathi, "Optimal placement and sizing of multiple distributed generating units in distribution networks by invasive weed optimization algorithm," Ain Shams Engineering Journal, vol. 7, no. 2, pp. 683-694, 2016.

[14] P. Ahmadi, M. H. Nazari, and S. H. Hosseinian, "Optimal resources planning of residential complex energy system in a day-ahead market based on invasive weed optimization algorithm," Engineering Technology \& Applied Science Research, vol. 5, no. 5, pp. 1934-1939, 2017. 


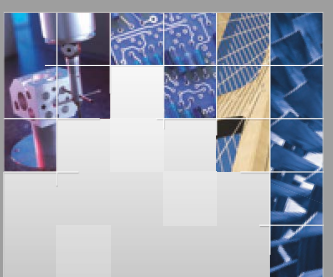

\section{Enfincering}
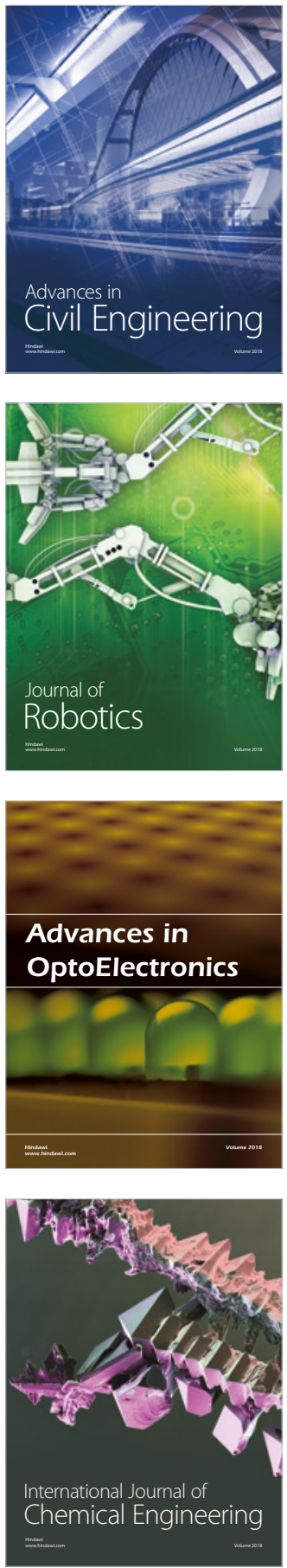

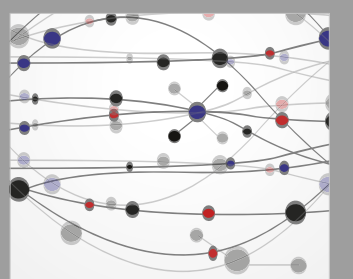

\section{Rotating \\ Machinery}

The Scientific World Journal

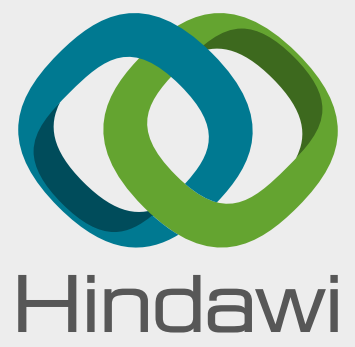

Submit your manuscripts at

www.hindawi.com
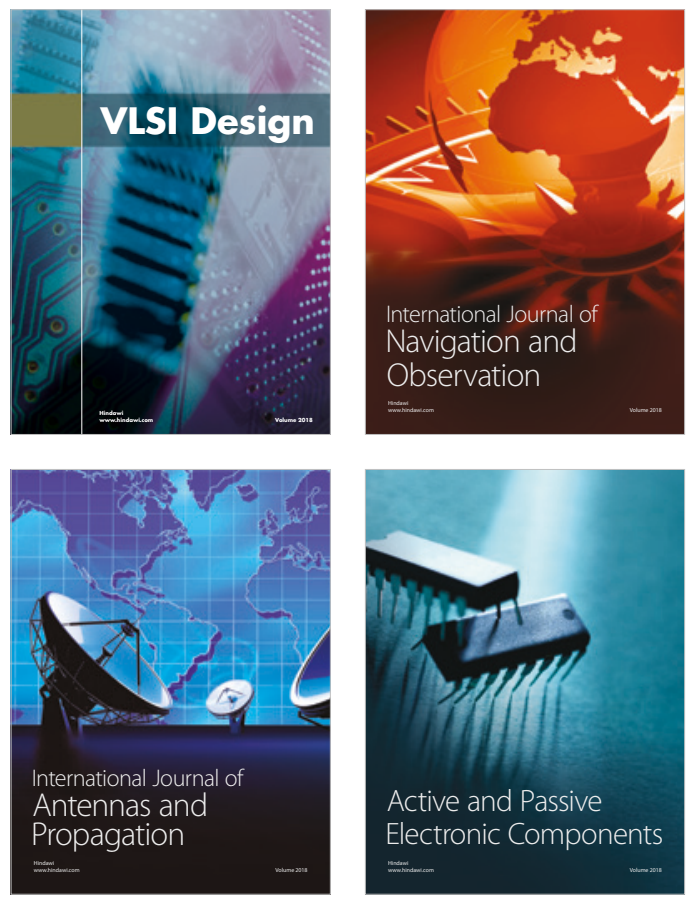
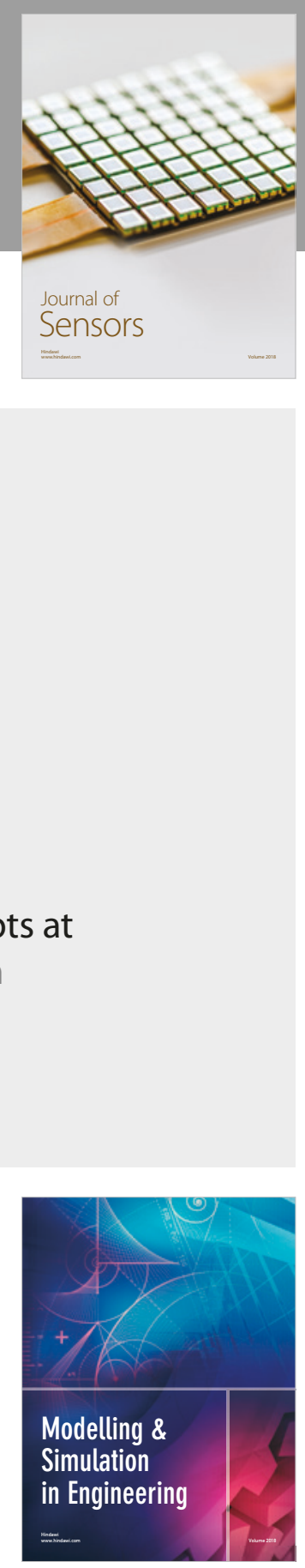

\section{Advances \\ Multimedia}
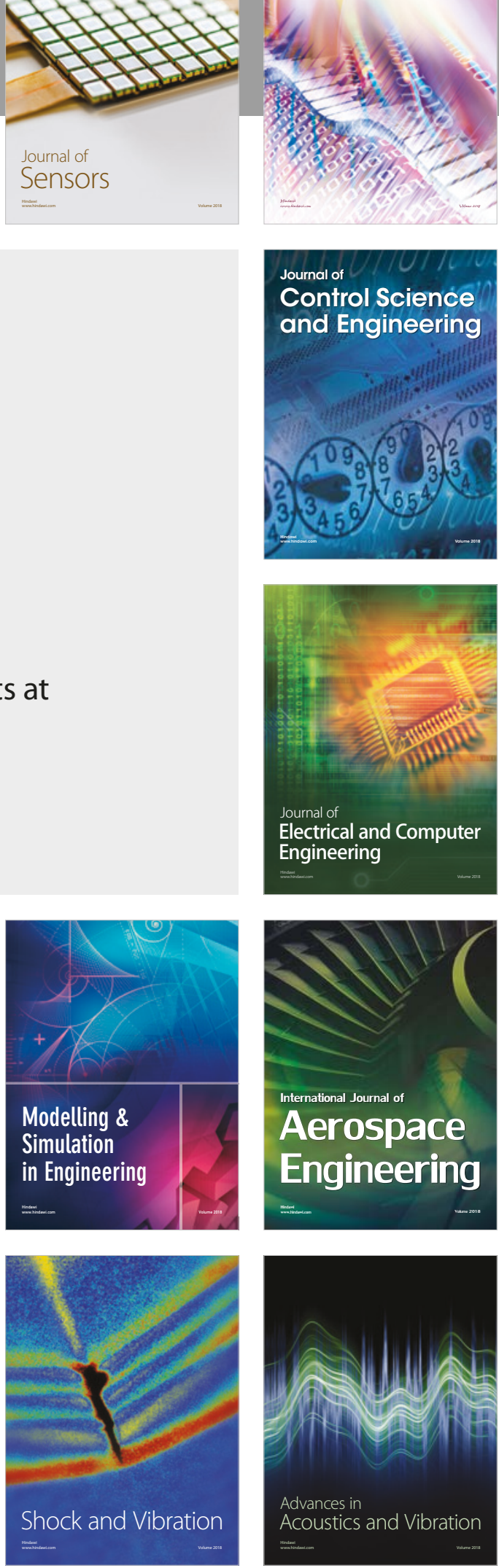